\title{
Three-dimensional photoacoustic imaging with a clinical two- dimensional matrix ultrasound transducer
}

Todd N. Erpelding, Yu Wang, Ladislav Jankovic, Zijian Guo, Jean-Luc Robert, et al.

Todd N. Erpelding, Yu Wang, Ladislav Jankovic, Zijian Guo, Jean-Luc Robert, Guillaume David, Chulhong Kim, Lihong V. Wang, "Threedimensional photoacoustic imaging with a clinical two-dimensional matrix ultrasound transducer," Proc. SPIE 7899, Photons Plus Ultrasound: Imaging and Sensing 2011, 78990A (17 February 2011); doi: 10.1117/12.875169 


\title{
Three-dimensional photoacoustic imaging with a clinical two- dimensional matrix ultrasound transducer
}

\author{
Todd N. Erpelding*a, Yu Wang ${ }^{\mathrm{b}}$, Ladislav Jankovic ${ }^{\mathrm{a}}$, Zijian Guo ${ }^{\mathrm{b}}$, Jean-Luc Robert ${ }^{\mathrm{a}}$, Guillaume \\ David $^{\mathrm{a}}$, Chulhong Kim ${ }^{\mathrm{b}}$, Lihong V. Wang ${ }^{\mathrm{b}}$ \\ ${ }^{a}$ Philips Research North America, 345 Scarborough Rd., Briarcliff Manor, NY, USA 10510; \\ ${ }^{b}$ Optical Imaging Laboratory, Dept. of Biomedical Engineering, Washington University in St. Louis, \\ 1 Brookings Dr., St. Louis, MO, USA 63130
}

\begin{abstract}
Photoacoustic tomography provides both structural and functional imaging in vivo based on optical absorption contrast. A novel imaging system that incorporates a two-dimensional matrix ultrasound probe for combined photoacoustic and ultrasonic three-dimensional (3D) volumetric imaging is presented. The system consists of a tunable dye laser pumped by a Nd:YAG laser, a commercial ultrasound imaging system (Philips iU22) with a two-dimensional matrix transducer (Philips X7-2, 2500 elements, 2-7 MHz), and a multichannel data acquisition system which allows us to acquire RF channel data. Compared with alternative 3D techniques, this system is attractive because it can generate co-registered 3D photoacoustic and ultrasound images without mechanical scanning. Moreover, the lateral resolution along the azimuth and elevational directions are measured to be $0.77 \pm 0.06 \mathrm{~mm}$ and $0.96 \pm 0.06 \mathrm{~mm}$, respectively, based on reconstructed photoacoustic images of phantoms containing individual human hairs. Finally, in vivo 3D photoacoustic sentinel lymph node mapping using methylene blue dye in a rat model is demonstrated.
\end{abstract}

Keywords: photoacoustic imaging, ultrasound, matrix ultrasound transducer, three-dimensional imaging, sentinel lymph node

\section{INTRODUCTION}

Photoacoustic imaging is a promising technology that combines image contrast based on optical absorption with ultrasonic resolution. The basis for photoacoustic wave generation is optical absorption by tissue structures that are illuminated by short pulses of light. Under stress and thermal confinement conditions, transient heating induces thermoelastic expansion and emission of acoustic pressure waves. These photoacoustic waves can be detected by conventional ultrasound array transducers for image reconstruction. Photoacoustic imaging overcomes the depth limitations of pure optical imaging techniques by utilizing ultrasonic detection, since acoustic scattering is 2-3 orders of magnitude less than optical scattering. It is capable of high-resolution structural, functional, and molecular imaging free from speckle artifacts. ${ }^{1}$

Recently, efforts have been made to incorporate two-dimensional (2D) array transducers for efficient three-dimensional (3D) photoacoustic imaging. Specifically, a 2D capacitive micromachined ultrasonic transducer (CMUT) with $16 \times 16$ transducer elements has been developed for photoacoustic and ultrasonic imaging. ${ }^{2}$ Mechanical scanning was used to simulate a larger 64 x64 element aperture for improved image quality. In addition, a 1.75D array transducer with 1280 elements $(10 \times 128)$ was constructed for ultrasonic and photoacoustic characterization of ex vivo ovarian tissue. ${ }^{3}$ Also, a polyvinylidene fluoride (PVDF) matrix array hydrophone with 590 transducer elements was used as a photoacoustic receiver in a research prototype system for breast cancer detection. ${ }^{4,5}$ The advantage of $2 \mathrm{D}$ arrays is that $3 \mathrm{D}$ images can be acquired without mechanical scanning and, by using a small number of laser firings, achieve higher imaging frame rates than other systems. The trade-off is increased system complexity to manage data from a larger number of transducer elements.

A promising clinical application of photoacoustic imaging is for noninvasive sentinel lymph node mapping. Sentinel lymph nodes are the first nodes to receive drainage from the tumor, and as such, are most likely to contain metastatic tumor cells. Biopsy of sentinel lymph nodes is an effective method to determine if the cancer has spread beyond the primary tumor into the lymphatic system. Sentinel lymph node biopsy is critical for cancer staging to plan appropriate

*todd.erpelding@philips.com

Photons Plus Ultrasound: Imaging and Sensing 2011, edited by Alexander A. Oraevsky, Lihong V. Wang, Proc. of SPIE Vol. 7899, 78990A - (C) 2011 SPIE · CCC code: 1605-7422/11/\$18 - doi: 10.1117/12.875169 
therapy and evaluate prognosis, and has become the standard of care for axillary staging in newly diagnosed breast cancer patients. ${ }^{6-8}$ The current method of sentinel lymph node biopsy depends on local injection of blue dyes and radioactive tracers that accumulate in sentinel nodes. Surgeons use incisions to invasively locate and excise sentinel nodes based on visual inspection for blue nodes and using Geiger counters to detect radioactive nodes. Excised nodes undergo pathological examination to determine the presence of metastases. A noninvasive method for sentinel lymph node identification could enable percutaneous needle biopsies as a viable option for axillary staging of breast cancer patients. The same blue dyes (i.e. methylene blue) currently used in clinical practice for sentinel lymph node biopsy can serve as photoacoustic contrast agents due to strong optical absorption by the dye. In vivo preclinical experiments in a rat model have successfully demonstrated noninvasive photoacoustic imaging of sentinel lymph nodes following injection of methylene blue, ${ }^{9}$ indocyanine green, ${ }^{10}$ gold nanoparticles, ${ }^{11,}{ }^{12}$ and carbon nanotubes. ${ }^{13}$ A modified clinical ultrasound system capable of photoacoustic imaging has highlighted the potential for clinical translation of photoacoustic sentinel lymph node mapping. ${ }^{14-16}$

In this report, the modified clinical ultrasound system has been extended to enable 3D photoacoustic and ultrasound imaging using a 2D matrix array transducer. This is the first time to our knowledge that 3D photoacoustic imaging has been demonstrated using a clinical 2D matrix array ultrasound transducer. The performance of this multi-modal 3D imaging system has been evaluated using resolution phantoms and for in vivo sentinel lymph node mapping.

\section{METHODOLOGY}

\subsection{Photoacoustic imaging system}

A prototype photoacoustic imaging system (Figure 1) has been built around a Philips iU22 ultrasound scanner (Philips Healthcare, Andover, MA). ${ }^{14-16}$ The modified channel board architecture allowed access to raw per-channel photoacoustic RF data, while retaining all imaging capabilities of an actual commercial ultrasound scanner. Raw photoacoustic and ultrasound RF data were transferred to a custom-built data acquisition system for post-processing image reconstruction, providing dual-modality imaging capability. The 2D matrix array probe X7-2 (2500 elements) with nominal bandwidth 2-7 MHz was used for 3D photoacoustic and ultrasound imaging. Currently, one photoacoustic volume image is generated from 36 lasers shots, requiring 20 seconds for data acquisition followed by offline image reconstruction using a 3D delay and sum beamforming algorithm implemented in Matlab (MathWorks Inc., Natick, MA). An FPGA-based electronic board and separate delay generator (Stanford Research Systems, Sunnyvale, CA) synchronized iU22 data acquisition with laser firings. The X7-2 transducer performs micro-beamforming within the transducer to reduce the number of system channels needed for the large number of transducer elements. The microbeamforming process performed within the transducer is reversed offline using a novel, proprietary algorithm to recover raw photoacoustic channel data. Photoacoustic maximum amplitude projection (MAP) images were reconstructed by first removing the skin signal and then projecting the remaining maximum signal amplitude along each A-line onto the corresponding $\mathrm{x}$ - y plane. Volumetric photoacoustic images were visualized by using VolView software (Kitware, Clifton Park, NY).

Light from a tunable dye laser (PrecisionScan-P, Sirah, Kaarst, Germany), pumped by a Q-switched Nd:YAG laser (PRO-350-10, Spectra-Physics, Santa Clara, CA) with 6.5-ns pulse duration and 10-Hz pulse repetition rate, was delivered via a fused end, bifurcated fiber bundle (LightGuideOptics, Rheinbach, Germany). Photoacoustic images were acquired using $650 \mathrm{~nm}$ light, which is close to the peak absorption wavelength for methylene blue dye. The light fluence on the skin was less than $10 \mathrm{~mJ} / \mathrm{cm}^{2}$, within American National Standards Institute safety limits. ${ }^{17}$

The fiber bundles were mounted to the matrix array probe to enable dual-modality $3 \mathrm{D}$ photoacoustic and ultrasonic imaging without mechanical scanning. The ultrasound probe was partially immersed in a water tank which had an opening at the bottom sealed with a thin, disposable, clear membrane. Rats were placed below the water tank, in contact with the membrane via ultrasonic coupling gel.

\subsection{Resolution phantom preparation}

To investigate the spatial resolution of the matrix probe, we imaged human hairs embedded within a gelatin phantom. A hot gelatin water solution ( $10 \%$ by weight) was made and congealed in mold. Two hairs were placed on top and another layer of gelatin was poured, completely covering the hairs. The gelatin phantom was placed in a water bath with the ultrasound transducer positioned $2 \mathrm{~cm}$ above the hairs. 


\subsection{Animal and dye preparation}

Animal protocols were approved by the Washington University Animal Studies Committee and animal handling was performed according to the guidelines on the care and use of laboratory animals. Healthy Sprague Dawley rats $(\mathrm{N}=3)$ were anesthetized using a mixture of ketamine $(85 \mathrm{mg} / \mathrm{kg})$ and xylazine $(15 \mathrm{mg} / \mathrm{kg})$. The hair in the left axillary region was gently depilated before imaging. Photoacoustic and ultrasound images were collected before and after $100 \mu \mathrm{L}$ intradermal injection of $1 \%$ methylene blue dye $(10 \mathrm{mg} / \mathrm{mL}$, American Reget, Inc., Shirley, NY) into the left forepaw pad. Photoacoustic images were collected at 5 minute intervals for up to 30 minutes post-injection, with an additional time point collected at $2.5 \mathrm{~min}$ post-injection. After imaging, animals were euthanized with an overdose of pentobarbital, and SLNs were dissected.

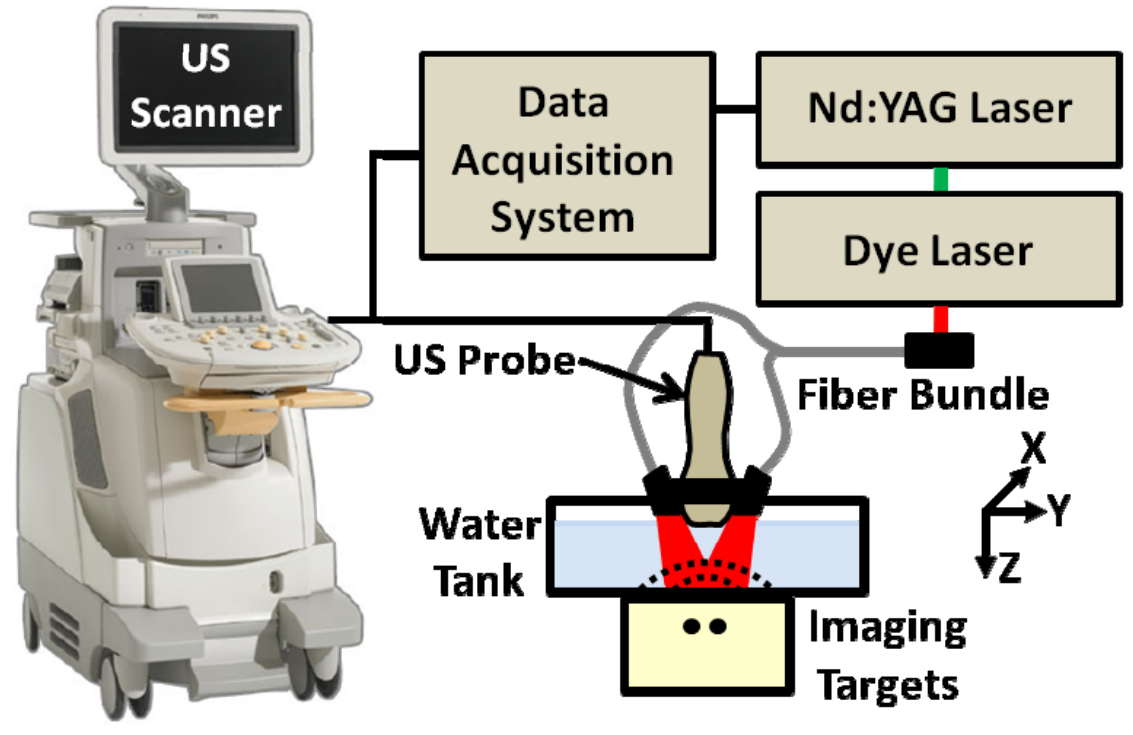

Figure 1. Experimental setup of the 3D photoacoustic imaging system adapted from a modified Philips iU22 ultrasound (US) imaging system (Philips Healthcare, Andover, MA).

\section{RESULTS}

\subsection{Resolution phantom imaging}

Photoacoustic images of two human hairs embedded within a gelatin phantom yield estimates of the spatial resolution of the matrix array transducer (Fig. 2). Figure 2a-b display photoacoustic maximum amplitude projection (MAP) images of the two hairs positioned along the azimuth and elevation direction, respectively. Figure 2c displays a photoacoustic Bmode image of the two hairs positioned along the elevation direction. Figure $2 \mathrm{~d}-\mathrm{f}$ show corresponding $1 \mathrm{D}$ line profiles as indicated by the dashed lines in Fig. 2a-c, respectively. Based on the measured full-width at half-maximum (FWHM) signal profiles, the average lateral resolutions along the azimuth and elevation directions are $0.77 \pm 0.06 \mathrm{~mm}$ and $0.96 \pm$ $0.06 \mathrm{~mm}$, respectively. The average axial resolution is $1.02 \pm 0.15 \mathrm{~mm}$.

\subsection{In vivo 3D photoacoustic SLN mapping}

Photoacoustic imaging with the matrix array transducer has been successfully demonstrated in vivo for 3D SLN mapping (Fig. 3). Photoacoustic MAP images show the accumulation of methylene blue dye in a rat SLN at 0, 5, and 30 minutes post-injection (Fig. $3 \mathrm{~b}, \mathrm{c}, \mathrm{d}$ ). The 0 minute time point was acquired immediately following methylene blue injection and shows a substantial increase in the mean photoacoustic signal amplitude from the SLN region compared with the control photoacoustic MAP image acquired prior to methylene blue injection (Fig. 3a). This signal increase reflects the rapid collection of methylene blue by lymphatic vessels in the rat forepaw and subsequent accumulation in the SLN. Since the methylene blue injection lasted about 10 seconds and 3D data acquisition took 20 seconds, the 0 minute time point consists of photoacoustic signals received up to 30 seconds after the start of the injection.

The dynamic increase in photoacoustic signal amplitudes from SLN regions following methylene blue injection is displayed according to mean and standard deviation (Fig. 3e). Photoacoustic signal amplitudes are plotted relative 

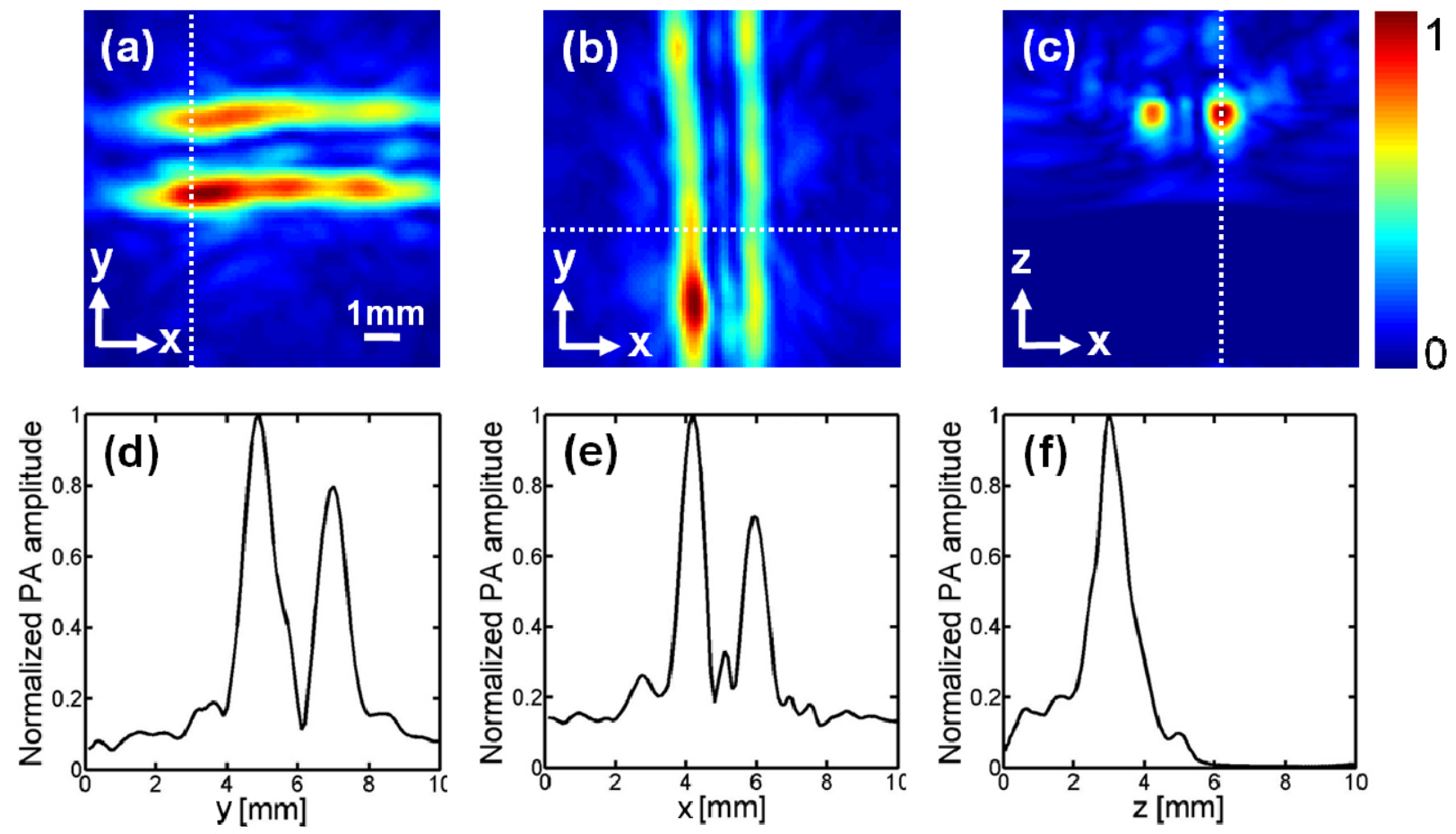

Figure 2. Photoacoustic images of two human hairs embedded within a clear gelatin phantom. (a) Photoacoustic maximum amplitude projection (MAP) image of hairs positioned along azimuth direction. (b) Photoacoustic MAP image of hairs positioned along elevation direction. (c) Photoacoustic B-mode image of hairs positioned along elevation direction. (d, e, f) 1D profiles along the dashed lines depicted in (a), (b), and (c), respectively.
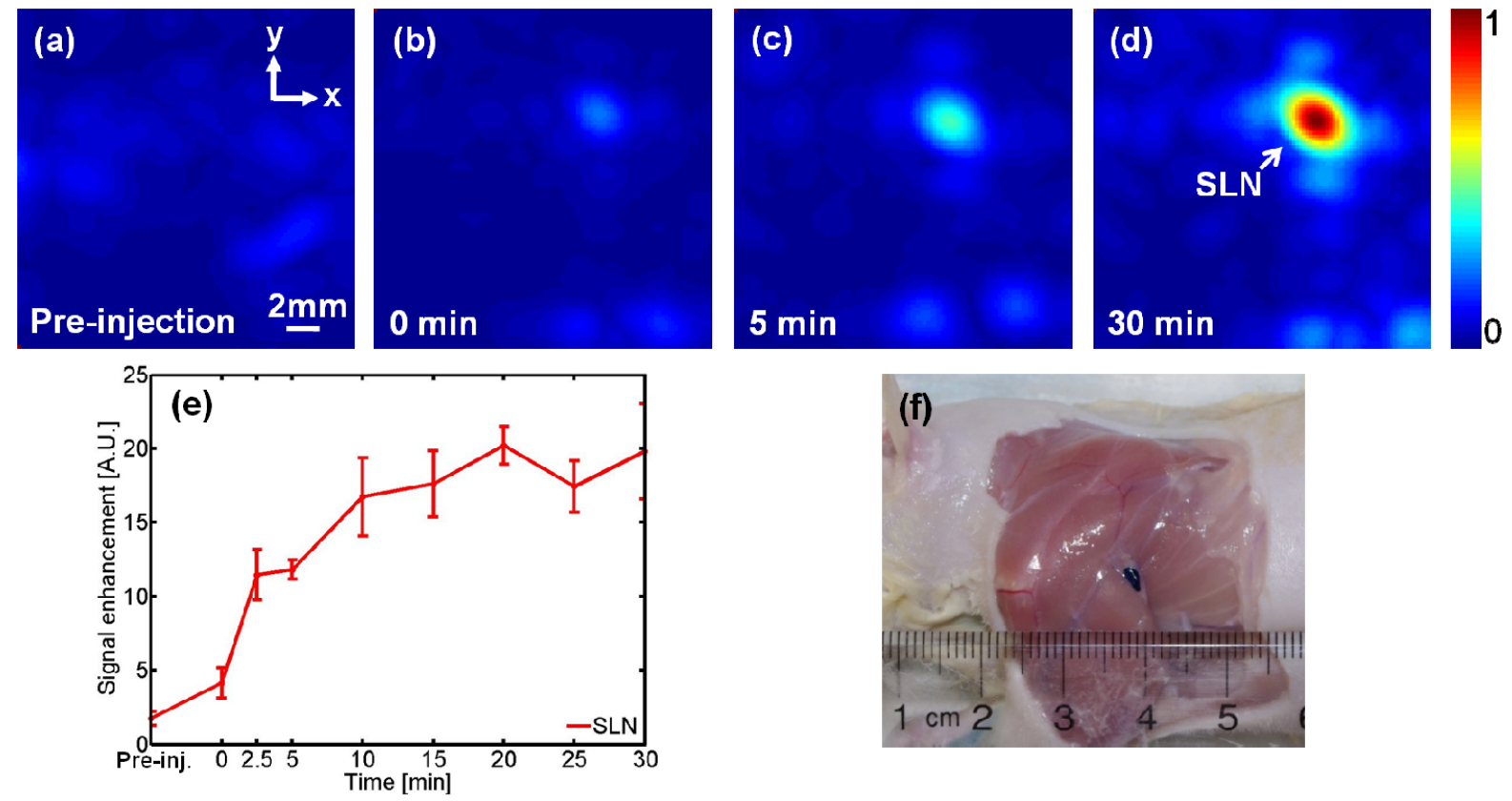

Figure 3. Noninvasive photoacoustic images of rat axillary region. (a) Control photoacoustic maximum amplitude projection (MAP) image collected prior to methylene blue injection into the rat forepaw. (b,c,d) Photoacoustic MAP images collected 0, 5, and 30 minutes following methylene blue injection, respectively, showing photoacoustic signal enhancement at the sentinel lymph node (SLN). (f) Graph shows dynamic changes in photoacoustic signal amplitudes from SLN. (f) Postmortem photograph of rat acquired after photoacoustic imaging and skin removal. 
to the pre-injection signal amplitude from the same region-of-interest. Photoacoustic signal amplitudes at 30 minutes post-injection increase approximately 20 times compared to the pre-injection baseline signal amplitudes, illustrating the strong contrast enhancement provided by methylene blue. The dynamics of methylene blue accumulation in a rat lymph node represented by the photoacoustic signal amplitude is consistent with previous reports, reaching peak signal amplitudes at 20-30 minutes post-injection. ${ }^{14}$ The accumulation of methylene blue dye in the rat SLN was confirmed by postmortem dissection (Fig. 3f).

Co-registered 3D photoacoustic and ultrasound images depict functional, represented by methylene blue accumulation, and structural information, represented by morphological features (Fig. 4). The location of the sentinel lymph node relative to the surrounding anatomy can be best appreciated in co-registered 3D photoacoustic and ultrasound images. Figure 4a illustrates a co-registered volume image with the indicated 2D B-mode slice depicted in Fig. 4b.
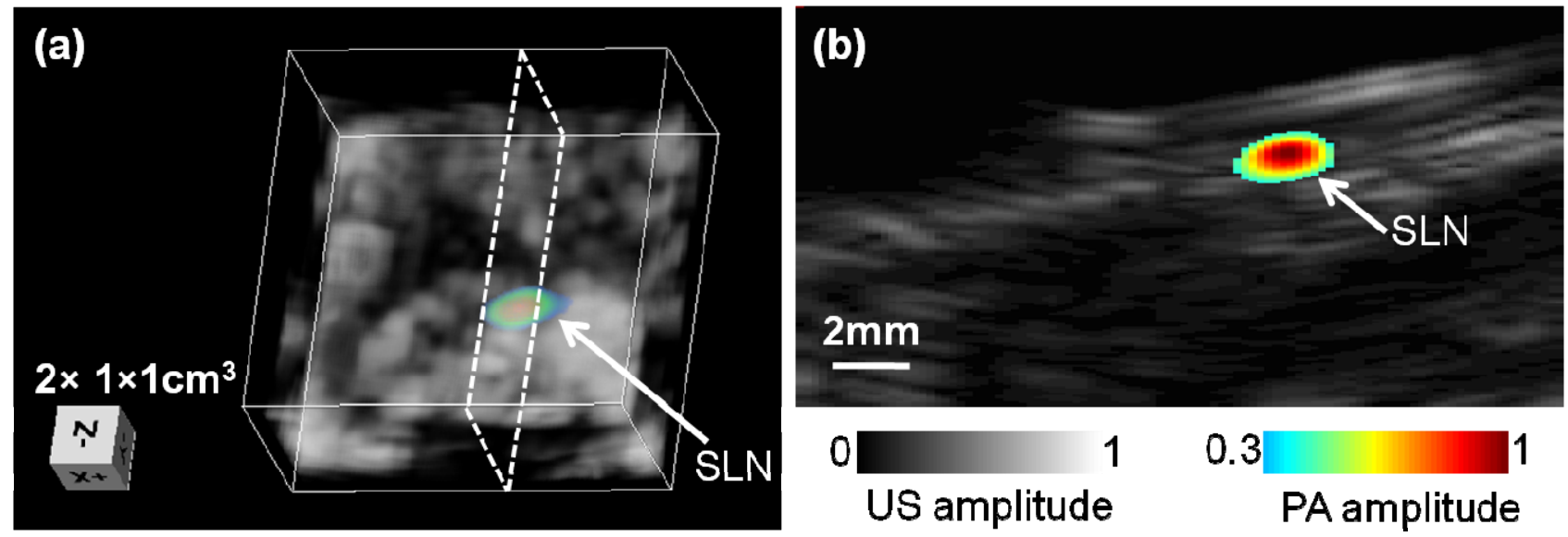

Figure 4. (a) Co-registered, in vivo 3D photoacoustic and ultrasound image acquired 30 minutes following methylene blue injection. (b) Co-registered photoacoustic and ultrasound B-mode image of rat sentinel lymph node (SLN). Photoacoustic signals are overlaid using a pseudo-color map.

\section{DISCUSSION}

A modified clinical ultrasound system has been adapted for 3D photoacoustic imaging using a 2D matrix array transducer. The addition of 3D photoacoustic imaging to this system provides an additional contrast mechanism based on optical absorption. The current system acquires a complete 3D photoacoustic dataset in 20 seconds from 36 laser firings and performs offline image reconstruction of a $4 \times 4 \times 5 \mathrm{~cm}^{3}$ volume. Compared to previous photoacoustic imaging systems developed around 2D matrix array transducers, ${ }^{2-5}$ this system offers fast photoacoustic data acquisition, submillimeter spatial resolution, and incorporates the full 3D ultrasound imaging functionality offered by the commercial iU22 system. The 3D ultrasound modes currently available on the system include live 3D imaging and live biplane imaging for real-time display of two planes simultaneously. Further system development could reduce the number of laser firings required and produce a photoacoustic volume image in real-time at a reasonable frame rate. Faster imaging frame rates will enable hand-held scanning, which is needed to fully realize the potential of $3 \mathrm{D}$ photoacoustic imaging with $2 \mathrm{D}$ matrix array transducers. The feasibility of 3D photoacoustic imaging using a modified clinical ultrasound imaging system has been established, which represents an important development towards this goal.

The results demonstrate the utility of the current system for dynamic 3D photoacoustic imaging in vivo. 3D photoacoustic images show the accumulation of methylene blue dye within a rat sentinel lymph node in the first 30 minutes following dye injection. Strong photoacoustic contrast enhancement $(\sim 20 \mathrm{x})$ resulted from methylene blue accumulation in the rat lymph node, which highlights the potential of this clinically-used dye. 3D photoacoustic imaging could offer unique clinical advantages for improved visualization of multiple sentinel nodes, as typically 1-3 nodes are removed during sentinel lymph node biopsy. Finally, percutaneous needle biopsies of sentinel lymph nodes could be guided more efficiently by $3 \mathrm{D}$ photoacoustic and ultrasound imaging than by $2 \mathrm{D}$ imaging alone. ${ }^{15,18,19}$ 


\section{ACKNOWLEDGEMENTS}

This research was supported by National Institutes of Health grants U54 CA136398 (Network for Translational Research), R01 EB000712, R01 NS46214, and R01 EB008085. LVW has a financial interest in Microphotoacoustics, Inc. and Endra, Inc., which, however, did not support this work. TNE, LJ, and JR are employees of Philips Research.

\section{REFERENCES}

[1] Wang, L. V., "Multiscale photoacoustic microscopy and computed tomography," Nat. Photonics 3(9), 503-509 (2009).

[2] Vaithilingam, S., Ma, T. J., Furukawa, Y., Wygant, I. O., Zhuang, X., De La Zerda, A., Oralkan, O., Kamaya, A., Gambhir, S. S., Jeffrey, R. B., Jr., and Khuri-Yakub, B. T., "Three-dimensional photoacoustic imaging using a two-dimensional CMUT array," IEEE Trans. Ultrason. Ferroelectr. Freq. Control 56(11), 2411-9 (2009).

[3] Aguirre, A., Guo, P., Gamelin, J., Yan, S., Sanders, M. M., Brewer, M., and Zhu, Q., "Coregistered three-dimensional ultrasound and photoacoustic imaging system for ovarian tissue characterization," J. Biomed. Opt. 14(5), 054014 (2009).

[4] Manohar, S., Vaartjes, S. E., van Hespen, J. C., Klaase, J. M., van den Engh, F. M., Steenbergen, W., and van Leeuwen, T. G., "Initial results of in vivo non-invasive cancer imaging in the human breast using near-infrared photoacoustics," Opt. Express. 15(19), 12277-85 (2007).

[5] Manohar, S., Kharine, A., van Hespen, J. C., Steenbergen, W., and van Leeuwen, T. G., "Photoacoustic mammography laboratory prototype: imaging of breast tissue phantoms," J. Biomed. Opt. 9(6), 1172-81 (2004).

[6] Ferrari, A., Rovera, F., Dionigi, P., Limonta, G., Marelli, M., Besana Ciani, I., Bianchi, V., Vanoli, C., and Dionigi, R., "Sentinel lymph node biopsy as the new standard of care in the surgical treatment for breast cancer," Expert review of anticancer therapy 6(10), 1503-15 (2006).

[7] Krag, D. N., Anderson, S. J., Julian, T. B., Brown, A. M., Harlow, S. P., Costantino, J. P., Ashikaga, T., Weaver, D. L., Mamounas, E. P., Jalovec, L. M., Frazier, T. G., Noyes, R. D., Robidoux, A., Scarth, H. M., and Wolmark, N., "Sentinellymph-node resection compared with conventional axillary-lymph-node dissection in clinically node-negative patients with breast cancer: overall survival findings from the NSABP B-32 randomised phase 3 trial," Lancet Oncol. 11(10), 927-33 (2010).

[8] Veronesi, U., Viale, G., Paganelli, G., Zurrida, S., Luini, A., Galimberti, V., Veronesi, P., Intra, M., Maisonneuve, P., Zucca, F., Gatti, G., Mazzarol, G., De Cicco, C., and Vezzoli, D., "Sentinel lymph node biopsy in breast cancer: ten-year results of a randomized controlled study," Ann. Surg. 251(4), 595-600 (2010).

[9] Song, K. H., Stein, E. W., Margenthaler, J. A., and Wang, L. V., "Noninvasive photoacoustic identification of sentinel lymph nodes containing methylene blue in vivo in a rat model," J. Biomed. Opt. 13(5), 054033 (2008).

[10] Kim, C., Song, K. H., Gao, F., and Wang, L. V., "Sentinel lymph nodes and lymphatic vessels: noninvasive dual-modality in vivo mapping by using indocyanine green in rats--volumetric spectroscopic photoacoustic imaging and planar fluorescence imaging," Radiology 255(2), 442-50 (2010).

[11] Song, K. H., Kim, C., Cobley, C. M., Xia, Y., and Wang, L. V., "Near-infrared gold nanocages as a new class of tracers for photoacoustic sentinel lymph node mapping on a rat model," Nano Lett. 9(1), 183-8 (2009).

[12] Pan, D., Pramanik, M., Senpan, A., Ghosh, S., Wickline, S. A., Wang, L. V., and Lanza, G. M., "Near infrared photoacoustic detection of sentinel lymph nodes with gold nanobeacons," Biomaterials 31(14), 4088-93 (2010).

[13] Pramanik, M., Song, K. H., Swierczewska, M., Green, D., Sitharaman, B., and Wang, L. V., "In vivo carbon nanotubeenhanced non-invasive photoacoustic mapping of the sentinel lymph node," Phys. Med. Biol. 54(11), 3291-301 (2009).

[14] Erpelding, T. N., Kim, C., Pramanik, M., Jankovic, L., Maslov, K., Guo, Z., Margenthaler, J. A., Pashley, M. D., and Wang, L. V., "Sentinel lymph nodes in the rat: noninvasive photoacoustic and US imaging with a clinical US system," Radiology 256(1), 102-10 (2010).

[15] Kim, C., Erpelding, T. N., Maslov, K., Jankovic, L., Akers, W. J., Song, L., Achilefu, S., Margenthaler, J. A., Pashley, M. D., and Wang, L. V., "Handheld array-based photoacoustic probe for guiding needle biopsy of sentinel lymph nodes," J. Biomed. Opt. 15(4), 046010 (2010).

[16] Kim, C., Erpelding, T. N., Jankovic, L., Pashley, M. D., and Wang, L. V., "Deeply penetrating in vivo photoacoustic imaging using a clinical ultrasound array system," Biomed. Opt. Express 1(1), 278-284 (2010).

[17] ANSI, [American national standard for the safe use of lasers. ANSI z136.1-2007] Laser Institute of America Orlando, Fla (2007).

[18] Downey, D. B., Fenster, A., and Williams, J. C., “Clinical utility of three-dimensional US,” Radiographics 20(2), 559-71 (2000).

[19] Su, J., Karpiouk, A., Wang, B., and Emelianov, S., "Photoacoustic imaging of clinical metal needles in tissue," J. Biomed. Opt. 15(2), 021309 (2010). 